\title{
Cesare Pavese: Il mestiere di poeta
}

\author{
LORENZO MONDO \\ La Stampa \\ lorenzo.mondo@libero.it
}

\section{Riassunto}

Percorso critico attraverso le due grandi raccolte di poesia di Cesare Pavese: Lavorare stanca e Verrà la morte e avrà i tuoi occhi.

Parole chiave: Poesia, Pavese.

Cesare Pavese: Il mestiere di poeta

\begin{abstract}
Critical jorney through the two major poetry compilations of Cesare Pavese: Lavorare stanca e Verrà la morte e avrà $i$ tuoi occhi.
\end{abstract}

Key words: Poetry, Pavese.

Mondo, Lorenzo. 2011. Cesare Pavese: Il mestiere di poeta. Cuadernos de Filología Italia$n a, \mathrm{n}^{\circ}$ extraordinario: págs. 257-267.

Cesare Pavese nasce poeta. È pacifico rifarsi ai versi che scrive a partire dai sedici anni, entrando in poetica "tenzone" con l'amico del cuore Mario Sturani. E prima ancora alla gran messe di poesie tenute nel cassetto e pubblicate postume. Siamo alla preistoria di Cesare, che rivela tuttavia nell'appassionata e cupa determinazione di farsi poeta, la forza di una vocazione. Anche se gli stessi amici, quelli appartenenti alla congrega del liceo D'Azeglio, gli renderanno pieno merito soltanto quando lo sentiranno recitare le lasse dei Mari del Sud. Quando, stando alla ben nota testimonianza di Massimo Mila, qualcuno avanzerà, senza l'ombra di un sorriso, il nome di Omero (Mila 1961). È la poesia che aprirà emblematicamente la raccolta di Lavorare stanca, che resterà a lungo il suo solo libro poetico. Ma sembrerebbe anche, tra tutti, il libro prediletto, sopravanzato appena nell'ultimo scorcio della sua vita dai Dialoghi con Leucò. Si badi alle dichiarazioni rilasciate in una lettera a Carlo Muscetta («Valgo più come poeta che come prosatore») e a Mario Alicata, 
dove si riconosce giusto il riferimento di Paesi tuoi a Lavorare stanca («che però vale di più»).

Sono lettere del 1941 e tengono indubbiamente conto del fervore di Pavese che, 1'8 giugno di quello stesso anno, propone a Giulio Einaudi, in tono scherzoso ma convinto, la pubblicazione di Lavorare stanca in edizione accresciuta e risistemata ( «Voi potete e dovete non lasciarVi sfuggire questa nuova occasione di rendere omaggio a un massimo poeta vivente...») (Pavese 2008). La nuova edizione uscirà nel 1943 e Cesare mostrerà in una lettera a Fernanda Pivano di esserne sempre innamorato: «Com'ero più mascalzone e intelligente a venticinque anni. Allora ho scritto un libro che nessuno stima un soldo, ma comunque non sarà più superato da nulla che io scriva». Ma ben più avanti nel tempo difenderà il suo primo libro con inusitato fervore: "Che senso ha dire che la poesia italiana moderna non esiste?...(Bada che io la difendo, questa poesia, anche senza tener conto che c'è uscito Lavorare stanca, libro che basta (non scherzo) a salvare una generazione» (lettera a Mario Motta del 23 gennaio 1950).

Ma torniamo ai Mari del Sud, che aprirà nel 1936 la silloge di Lavorare stanca. Il 27 maggio di quell'anno, Leone Ginzburg scrive a Carlo Muscetta una lettera in cui l'apprezzamento per Pavese viene rafforzato da un inatteso accostamento: «Ritengo Lavorare stanca il più bel libro di versi uscito in Italia a rivelare un poeta nuovo dopo La via del rifugio. Questo curioso Piemonte!» (Ginzburg 2004). Non è un omaggio da poco quando si pensi che nelle edizioni di Gobetti hanno visto la luce, fin dal 1925, gli Ossi di seppia di Montale, non insensibile per parte sua al fascino di Gozzano. Ma stupisce nelle parole di Ginzburg l'accoppiata Gozzano-Pavese. E se l'ammirazione per Cesare si spiega con il fatto di aver visto nascere le sue poesie e averle tenute a battesimo insieme con la "confraternità" del D'Azeglio, quella per Gozzano ubbidisce a un'altra, non partecipata e sfuggente misura. Si direbbe comunque che Ginzburg non condivida lo stereotipo, che sarà divulgato dai suoi sodali e seguaci, di un Gozzano chiamato a rappresentare una stagione di separatezza e rassegnazione civile, una Torino sfinita e appunto crepuscolare che avrebbe ripreso vigore con la combattiva presenza gramsciana e gobettiana. Come se il giellista Ginzburg, sottraendosi ad artificiosi anacronismi, riconoscesse in Gozzano, insieme al dono della poesia, il valore corroborante dell'ironia. Quanto al confronto con Pavese, nasce forse da una valutazione estemporanea ma piace immaginare che alle sue sensibilissime antenne non sia sfuggito il sotterraneo legame tra i due poeti. È possibile poi che, frequentando Cesare, abbia conosciuto la sua familiarità con il poeta di Agliè.

La lettera di Ginzburg mi porta a rievocare la piccola scoperta che credetti di fare in un saggio pubblicato molti anni fa (Mondo 1964; 1969). Mi accadde infatti di leggere una poesia di Pavese intitolata Frasi all'innamorata, allora inedita e datata nella stesura definitiva 4-10 agosto 1930. Scritta dopo mesi di silenzio, rappresenta uno stacco netto rispetto alle poesie precedenti, le poesie-sfogo, quelle che gli amici

Cfr. Centro Studi Guido Gozzano-Cesare Pavese dll'Università di Torino, Collocazione APX52, Scritti giovanili, c. 155. 
definivano «del tormento», e manifesta un maggior controllo dei sentimenti, esibendo il verso lungo di 13, 14 sillabe che sarà tipico di Lavorare stanca:

Ecco il Pò. - 'Com'è bello... Stasera è un cristallo.

'Le colonne di luce e la curva del molo:

pare quasi, nel buio, la spiaggia del mare.

La compagna mi parla gioiosa e mi stringe:

dovrò anch'io abbracciarla più stretto sul ponte.

Un'orchestra lontana c'insegue fin qui.

Le colline son buie. - 'Verresti in collina?'

'No, in collina. È lontano. Restiamo a guardare'.... (Pavese 1998: 297-298).

Insisto ad avvertire nella poesia una forte suggestione gozzaniana, quella che si poteva cogliere solo per labili indizi in altri luoghi pavesiani, talora d'impronta parodistica e goliardica. Colpisce, sia pure in un diverso contesto ambientale e comportamentale, certa assonanza con La signorina Felicita. Analoga è la situazione, analogo il ritegno della signorina di città e della signorina di campagna davanti a una possibile compromissione con l'accompagnatore. Felicita, dopo avere indugiato a numerar le stelle insieme all'Avvocato, alter ego di Gozzano, si riscuote dal paesistico, galeotto incanto, quasi temendo di non padroneggiare i propri sentimenti: «Scendiamo! È tardi: possono pensare / che noi si faccia cose poco belle...». La spregiudicata ragazza di Pavese non teme la riprovazione per essere scesa con l'amico, di sera, in riva al Pò, ma conserva qualche scrupolo, se reagisce alle insistenze di lui che vorrebbe portarla in barca sul fiume, farle varcare il confine di un malcerto rapporto: «Di sotto il caschetto mi guarda / e poi, quasi compunta, ripete/ 'Restiamo a guardare...».

Il caschetto di feltro denota tra l'altro una attenzione per l'abbigliamento femminile che è tipica di Gozzano e che il poeta canavesano esprimerà, in un'altra occasione, cittadina e mondana, con l'emblematica «veletta». Ma più flagrante è il rapporto tra i due poeti segnalato dall'incipit che troviamo in un primo abbozzo di Frasi all'innamorata: «Passeggiamo in silenzio con una bambina/abbordata per strada, lungo il viale, di sera...», che rinvia con ogni evidenza alla squilla Mari del Sud, con cui s'inaugura Lavorare stanca: «Camminiamo una sera sul fianco di un colle,/in silenzio...» (conta anche la data di questa poesia, 7-19 settembre-novembre 1930, a breve distanza, cioè da Frasi all'innamorata. Sono indizi che lasciano intravedere anche una amicizia stilistica, il suggerimento da parte di Gozzano di un verso narrativo e prosastico che in Frasi all'innamorata, ma non soltanto, si apre alle mosse vivaci del dialogo.

Certo il verso pavesiano, che abolisce il cantato dell'endecasillabo e abbandona gli stessi residui di rima interna, diventa altra cosa, si appoggia ad altre esperienze, quelle della poesia americana, soprattutto di Whitman. Ma Frasi all'innamorata sta lì a dimostrare, nel suo sostanziale isolamento, il pagamento di un debito e insieme la rimozione da parte di Pavese d'una significativa occasione-spinta sul modo di fare poesia. 
Mi conforta nell'idea uno studio di Anco Marzio Mutterle secondo cui il poeta canavesano:

sembra porsi in qualità di presenza familiare e forse proprio in ragione di ciò rimossa, di consuetudine che si fa lampante nei momenti iniziali e terminali della sua poesia, fino a Verrà la morte e avrà i tuoi occhi, quando cioè il sistema espressivo lascia trasparire il numero più alto di incrinature e scollamenti, tra i quali si insinuano il riecheggiamento e magari la memoria involontaria, anche nei riguardi di D’Annunzio e Leopardi (Mutterle 1987: 160-161).

La mia insistenza sull'argomento non deriva da un puntiglio esegetico, ma vale a segnalare un comportamento tipico di Pavese, il confronto e l'abbandono pressochè subitaneo nei riguardi di autori amati o congeniali per foggiarsi un personalissimo strumento espressivo. Perchè I mari del Sud, con la sua larga apertura verso il futuro, sembra comportare anche la chiusura verso un certo passato, quanto meno l'accantonamento di un esplicito gozzanismo (che riemergerà in marginali e inopinati anfratti).

Così, conta per Pavese, come suggerimento e stimolo, la lezione di Whitman, l'ammiratissimo poeta delle Foglie d'erba al quale ha dedicato la tesi di laurea. Gli deve senza dubbio, per sua esplicita ammissione, la scoperta del volgare americano e l'uso del dialetto piemontese per rinsanguare la lingua della tradizione letteraria italiana che gli appare estenuata. Ammira il suo verso libero, ma ne prende le distanze affermando: «Mi mancava insieme il fiato e il temperamento per servirmene». Il verso lungo dei Mari del Sud sarebbe nato invece da una certa «tiritera», istintiva e casuale, che si sarebbe trovato a modulare nel comporre la poesia. Un verso lungo perchè «sentivo di aver molto da dire e di non dovermi fermare a una ragione musicale nei miei versi, ma soddisfarne altresì una logica». Ora, pur proteggendosi dall'enfasi declamatoria di Whitman, dalla sua propensione al catalogo tematico, è ben lui a riconoscere nel poeta americano la suggestione di un verso che «è un tutto finito, con le sue armonie e il suo significato». Storia nota, anche se non è da sopravvalutare in Pavese l'importanza di Whitman e, più in generale, della letteratura d'oltreoceano. Ad esempio, per quanto riguarda il movimento del verso, non riconducibile nella sua tenuta a quell'accennato mugolìo, si è ritenuto plausibilmente che risenta di una impostazione classica, che conservi memoria di una certa tradizione metrica italiana (Riposio 1987).

Escludendo con questo anche la fantasiosa ipotesi di Massimo Mila, che ravvisava nella poesia epico-narrativa di Pavese, «ricca di personaggi e racconti», con il suo dispiegato ritmo ternario, una ascendenza etnica e culturale: quella esemplata dai Canti popolari del Piemonte raccolti da Costantino Nigra (Mila 1961). Un altro canavesano illustre, diplomatico di razza ed esploratore del substrato celtico nella poesia popolare dell'Italia settentrionale. Piacerebbe arruolarlo accanto a Gozzano tra gli antecedenti di Pavese, indurre il nostro poeta a respirare anche per questa via l'aria del bel Canavese. Ma ne accenno soltanto per rammentare a quali discordanti esiti abbia portato la vexata quaestio della tecnica poetica pavesiana. 
Altro problema è rappresentato dalla struttura di Lavorare stanca, e segnatamente di quella evidenziata dall'edizione definitiva del 1943. Pavese, lo sappiamo, si è soffermato a lungo sul problema. Si è chiesto se fosse possibile rinvenire tra poesia e poesia un legame fantastico o concettuale, se egli avesse cioè realizzato un canzoniere. E qui, a suggestionarlo, sulla scorta dei maestri torinesi - da Ferdinando Neri a Luigi Foscolo Benedetto - è l'architettura delle Fleurs du mal. Baudelaire, oltre a un certo maledettismo d'impronta urbana, gli forniva l'esempio di una poesia narrativa, scandita da capitoli con diversa titolazione. È da notare che, ispirandosi a una consuetudine d'oltralpe, un francesista italiano abbia incluso tranquillamente le poesie di Baudelaire in una silloge di narratori (Bogliolo 1991). Pavese negherà di essere riuscito in analoga impresa. «Tutt'al più, come nell'Alcione, si tratta di un legame temporale, fantasie da giugno a settembre».

La costruzione, semmai, agirebbe all'interno di ogni singolo componimento, che vuole essere appunto una poesia-racconto. Eppure, all'idea del canzoniere sembrerebbe avere rinunciato con fatica. Già nelle ' 45 poesie che compongono la prima edizione di Lavorare stanca, quella del 1936, si coglie infatti qualche cenno di un possibile raggruppamento che non si curi dell'ordine cronologico. Il tentativo sembra abortito sul nascere, e la raccolta risente d'altronde dello scompiglio provocato dalle poesie espulse a causa della censura e da quelle aggiunte in extremis nell'esilio di Brancaleone. Ma è significativo che nella seconda edizione del libro, quella del 1943, che si avvale delle poesie recuperate e di quelle composte successivamente (per un totale di 70 componimenti) proceda a dividerlo in sei gruppi o sezioni. Queste dovrebbero essere caratterizzate da una concordanza tematica segnalata dai titoli. Un risultato che risulterà, alla fine, abbastanza approssimativo, ma che, se non mi sbaglio, appare comunque ricco di implicazioni.

Fin qui, gli echi più vistosi che si possono rintracciare delle sue letture. Ma altri, più occulti e capziosi, la critica va scoprendo, da Leopardi a D'Annunzio a Montale, fino all'insospettabile Verlaine rilevato da Bàrberi Squarotti nella poesia Poggio Reale (Bàrberi-Squarotti 2001). Non viene intaccata ovviamente l'originalità di Lavorare stanca, di quella che lui stesso, nella fascetta editoriale del 1943, definirà «una delle voci più isolate della poesia contemporanea». Non viene compromessa la tenuta di un libro pressochè assoluto e totalizzante, tale da rappresentare un sostanziale congedo dal fare poetico. Lavorare stanca, semenzaio di situazioni e figure che compariranno nella narrativa, è un libro compatto pur nelle sue oscillazioni tematiche e stilistiche, nel movimento in cui si configura l'accidentata storia di un'anima. Così la sua poesia che voleva essere muscolosa e oggettiva finisce per accogliere il ripiegamento sull'io e sugli affioramenti interiori. Diventa, da realistica, simbolica. Il suo andamento severo, spesso di tipo sapienziale, cede a una più veloce e musicale scansione, fino ad acquistare la patina dell'un tempo vituperata poesia pura. Così: «Non ci sono ricordi su questo viso./ Solo un'ombra fuggevole, come di nube./ L'ombra è umida e dolce come la sabbia/di una cavità intatta, sotto il crepuscolo./ Non ci sono ricordi. Solo un sussurro/ Che è la voce del mare fatta ricordo». Ma proviamo a esaminare le emergenze tematiche di Lavorare stanca, tenendo conto delle partizioni proposte da Pavese nell'edizione definitiva. Precisando e arricchendo eventualmente le chiose dettate a suo tempo da Italo Calvino, il primo a essersi 
cimentato sul tema (Pavese 1962). La sezione intitolata Antenati è tra le più compatte. Celebra gli antenati che vanno considerati in senso estensivo, a partire da un autobiografismo indicato anche dai verbi in prima persona. C'è il favoloso cugino dei Mari del Sud che, dopo avere errato per il mondo fino a diventare cacciatore di balene, sente il richiamo irresistibile della terra natì, perchè «Le Langhe non si perdono». È un'affermazione che, così perentoria, lascia la sua impronta, finisce col permeare l'intera sezione. Tanto più che viene fatta propria dal ragazzo Pavese: «...e io penso alla forza/che mi ha reso quest'uomo strappandolo al mare». Dice bene Pavese: «mi ha reso quest'uomo», come se la forza misteriosa volesse istituire un rapporto con lui, sceglierlo quale interlocutore privilegiato.

Tra gli ascendenti familiari, ne figura uno che disattende il suo lavoro in negozio per leggere romanzi, che è attratto più generalmente dall'ozio contemplativo: «Siamo nati per girovagare su quelle colline,/ senza donne, e le mani tenercele dietro la schiena», (dove il «senza donne» riassume, nella poesia, una serie di tirate misogine). Vengono tuttavia assunti al mondo degli antenati anche i villani, e il termine acculturato che li designa già comporta il riconoscimento di una certa dignità. Pur nell'aspra fatica, appaiono liberi e scanzonati, esibiscono il piglio di minime divinità terragne, quasi di domestici lari. Al ritorno dai campi, «Sogghignano ai gruppi di donne/o domandano quando, vestite di pelle di capra,/ siederanno su tante colline a annerirsi nel sole» (e le pelli di capra sembrano contenere un presagio di dionisiache apparizioni). Questo non impedisce che il sole rovente possa schiantarli al suolo, che inveiscano contro la brina perchè distrugge il grano. $\mathrm{O}$ che si appostino nella vigna per difenderne grettamente i frutti -la «roba»- dai vagabondi. È un'altra opzione, aliena da poetizzanti sovrapposizioni, che Pavese si concede nella sua tipica ambivalenza. Del resto, è la terra a esercitare su tutto la sua ancestrale signoria, quasi di suprema genitrice e antenata. «Vedo solo colline e mi riempiono il cielo e la terra/ con le linee sicure dei fianchi, lontane o vicine». Per il bambino Pavese la campagna è un paese «di verdi misteri», memore del baudelairiano «vert paradis des amours enfantines». Nel quale possono aprirsi notti di tregenda, affermarsi mitiche apparizioni, sulle peste del nero caprone che incendia le colline di violenza e lussuria. Il sentimento complessivo del poeta sembra essere, se non di rimpianto, di oscura fascinazione. E a chiudere la parabola avviata dai Mari del Sud è La notte, dove non a caso riaffiora l'infanzia dello scrittore. Non più segnata tuttavia dai liberi, e avventurosi, giochi infantili ( $(\mathrm{OH}$ da quando ho giocato ai pirati malesi,/ quanto tempo è trascorso...») ma da un presagio di cosmico smarrimento:

Per la vuota finestra

il bambino guardava la notte sui colli

freschi e neri, e stupiva di trovarli ammassati:

vaga e limpida immobilità. Fra le foglie

che stormivano al buio, apparivano i colli

dove tutte le cose del giorno, le coste

e le piante e le vigne, eran nitide e morte

e la vita era un'altra, di vento, di cielo,

e di foglie e di nulla. 
Sono versi che, con quella cadenza sul «nulla», hanno fatto pensare giustamente a Leopardi (Pavese 1998). Ma La notte è importante anche perchè sottolinea il passaggio dalla poesia-racconto dell'esordio alla seconda maniera di Pavese, più incorporea ed enigmatica. In questa prima sezione, dunque, Pavese sembra inseguire un coerente, dinamico disegno morale e stilistico.

Le poesie intitolate Dopo trovano una sostanziale unità nel tema dell'amore: adolescenziale e virile, atteso e realizzato, condiviso e subito, talora orgogliosamente eluso come in Mania di solitudine: «Il quadrato di cielo/ mi susurra di tutti i fragori, e una stella minuta/ si dibatte nel vuoto, lontano dai cibi,/ dalle case, diversa. Non basta a se stessa,/ e ha bisogno di troppe compagne. Qui al buio, da solo,/ il mio corpo è tranquillo e si sente padrone». Dove si affaccia anche, con forza, l'immagine ricorrente della finestra, come risicata apertura al mondo, come separatezza che non esclude spiragli di evasione.

L'amore è vissuto dalla parte dell'uomo o della donna, può essere mercenario eppure gioioso, non privo di compassione e tenerezza, come accade in Tolleranza o La puttana contadina; più sovente è un amore delicato ed esclusivo, fonte di inauditi stupori, di identificazione dell'amata con quanto il poeta ha di più caro. «Hai nel viso un silenzio che preme il cuore/ con un tonfo, e ne stilla una pena antica/ come il succo dei frutti caduti allora». Ma come si spiega il titolo della sezione, che riprende quello di una poesia posta in chiusura? Parrebbe dovuto a una motivazione di natura intima, esistenziale, al ricordo di una intensa, speciale emozione. Alla poesia Dopo, Pavese teneva particolarmente, come risulta in due lettere inviate dal confino alla sorella Maria. Arriva a dire parlando di quei versi: « ...è la mia sola compagnia, perchè non penso ad altro». Siamo al 19 agosto 1935 e al 2 ottobre prega la sorella di riferire a Tina che di tutte le sue poesie «vale qualcosa soltanto quella intitolata». Chiuso nella sua stanza, appunto «dopo un convegno d'amore con Tina, Cesare immagina che la gioia e il calore vitale di due amanti si irradi lungo i viali e le strade della città, che tutto sia partecipe dei loro sentimenti. Una poesia che, al di là di ogni successivo sconforto, sembra esprimere un fiducioso vaticinio e per questo viene privilegiata.

Città in campagna è la terza sezione del libro. Riesce inevitabile citare, con le parole di Pavese, quella che sarebbe stata l'ispirazione prima di Lavorare stanca: cioè «l'avventura dell'adolescente che, orgoglioso della sua campagna, immagina consimile la città, ma vi trova la solitudine e vi rimedia col sesso e la passione che servono soltanto a sradicarlo e gettarlo lontano da campagna e città, in una più tragica solitudine che è la fine dell'adolescenza» (Pavese 1943). In queste poesie l'apparente contrasto tra città e campagna si risolve in una sorta di mutua compenetrazione e compensazione, non soltanto figurale ma etica. La città è vista per lo più con gli occhi del contadino o di chi ne è comunque emarginato: il barbone che si aggira sui marciapiedi, smarrito per la penuria di cibo e di vino; la ragazza lavoratrice che torna in treno al paese con una voglia esitante di luci e di vetrine; i sabbiatori che il fiume riconduce dopo la dura fatica ai lumi scintillanti di Torino. La città è il miraggio di chi ne è fuori e arriva a scoprire le sue lusinghe e i suoi inganni. È un luogo di avventura, e ad essa vanno assegnate idealmente le fughe dei ragazzi fuori di casa, gli ulissidi protesi alla scoperta di sè e del mondo. Pavese simpatizza con loro: «...il 
ragazzo cammina/ tutto il giorno per strada. Non cerca ancor donne/ e non gioca più in terra. Ogni volta ritorna./ Il ragazzo ha un suo modo di uscire di casa/ che, chi resta, s'accorge di non farci più nulla». In realtà Pavese, che non è più un ragazzo, non soltanto vorrebbe tornare ogni giorno a casa, ma trovarci una donna. Come recita, concludendo questa sezione, la poesia intitolata Lavorare stanca. Come già nella sezione Dopo, Pavese sembra attribuire al finale uno speciale significato, correttivo e compensativo.

Maternità come ha visto Calvino, si caratterizza sostanzialmente per la «rabbia sessuale» e il «senso carnale della generazione dei figli». Le calde, sensuali attrazioni femminili non risparmiano i morti, che si riaggirano come fantasmi notturni posseduti da un «ansia inesausta/ di contatti e sapori». Ma la donna viene poi assimilata alla terra, con il suo destino di madre votata alle generazioni, mentre il corpo dell'uomo è capace di compiere «tante altre cose». Si pensa all'asserzione della poesia Antenati: «...e le donne non contano nella famiglia». La donna, in quanto tale, appartiene al mondo dei sacrificati ed esclusi. Ma la brutalità, l'apparente cinismo con il quale viene a momenti tratteggiata, sembra corretto dalla poesia Maternità, che forse non a caso presta il suo titolo alla sezione. La protagonista risulta assente, è morta. Qualcosa della sua vita è rimasta soltanto, senza che essi lo sappiano, negli occhi dei figli. E il padre non sa più ritrovarla, come non si riconosce nei figli, spavaldi e diversi da sè. Questa donna, un tempo, «era giovane/ e rideva e parlava, ma è un gioco rischioso/ prender parte alla vita». È eccessivo pensare a un ripensamento virtuoso, ma certo va segnalata questa notazione pietosa in un gruppo di poesie assegnabili alla passione rovente e istintuale: agli impulsi che rischiano di fomentare - secondo le parole di Pavese - una «più tragica solitudine».

Le sette poesie di Legna verde compongono il gruppo più coeso della raccolta $\mathrm{e}$, tenuto conto dei tempi, rappresentano un esempio pressochè unico di poesia ispirata alla politica e alla storia. Si comincia con la rassegnazione degli operai che, dopo la pausa del pranzo, torneranno al lavoro «come un gregge svogliato». Si continua con Fumatori di carta, dove il suonatore di clarino infila nelle note la sua acuta protesta contro l'ingiustizia, la sua ansia di riscatto sociale: «...non era il destino se il mondo soffriva,/ se la luce del sole strappava bestemmie:/ era l'uomo, colpevole». Ma poi nella città si sentono echeggiare spari, al mattino si cancellano sul selciato le macchie di sangue, «l'uomo solo» (un leitmotiv che cadenzerà alcune delle più belle poesie pavesiane) ripensa ai compagni che stanno in prigione. A lui stesso toccherà contemplare, dalla finestra di un carcere, il passaggio quasi prodigioso, come segnacolo di libertà, di «una nube soda e bianca, che indugia nel quadrato del cielo».

Fumatori di carta e Legna verde sono titoli che danno una idea di impotenza e incompiutezza nei confronti della tirannia e della persecuzione, ribadiscono in termini lapidari, vagamente proverbiali, il senso delle poesie. Fin qui abbiamo avuto comunque una progressione, il delinearsi di una storia che coinvolge fisicamente Pavese. Ma ecco affacciarsi al termine della sezione i versi enigmatici di Parole del politico. Il poeta, che si rifà ai giorni in cui era confinato a Brancaleone, indugia con un amico al mercato del pesce. Ma il loro colloquio resta inespresso, segnalato appe- 
na dal titolo oscuramente allusivo. Non si può fare a meno di confrontare questa afasia con la veemenza protestataria di Nuto, il suonatore di clarino. Sembra di avvertire allora, nel mutismo imposto da Pavese al compagno di pena, il suo disincanto nei riguardi della politica. Come se non volesse più sentirne parlare. Nella poesia contano soltanto i colori iridati dei pesci (e più avanti le movenze sensuose delle donne) che esprimono una urgenza vitale, la speranza di un imminente ritorno alla libertà: «Al confronto col mare tutto scaglie d'argento,/ la vincevano i pesci. Si pensava al ritorno».

Paternità è il titolo, non esaustivo, di un raggruppamento abbastanza composito. Ma trova il suo fuoco centrale nella poesia omonima. Analogamente a ciò che accadeva con Maternità, si direbbe che Pavese abbia qualche resipiscenza, voglia fare ammenda di certe crudezze. Qui il tema della solitudine, che sembra l'approdo ultimo di Lavorare stanca, si intormentisce alla vista dei bambini che giocano sulla riva del mare, lasciando trasparire un desiderio sofferto di paternità. Cala la notte e "l'uomo solo» torna a riflettere sulla sua privazione: «Ci son donne a quest'ora che spogliano un bimbo/ e lo fanno dormire». Viene in mente il rapporto speciale con l'infanzia che Pavese manifesta in certi romanzi, soprattutto con la figura di Dino nella Casa in collina. La solitudine detta a Pavese alcune delle poesie più intense, in cui la sorte dell'uomo si misura con quella delle parvenze naturali: «Non c'è cosa più amara che l'alba di un giorno/ in cui nulla accadrà. Non c'è cosa più amara/ che l'inutilità. Pende stanca nel cielo/ una stella verdognola, sorpresa dall'alba». Il tema lambisce anche la poesia Mito, che anticipa i Dialoghi con Leucò, per quanto riguarda il crepuscolo degli dei, l'amara consapevolezza dell'irrecuperabile infanzia del mondo: «Verrà il giorno che il giovane dio sarà un uomo,/ senza pena, col morto sorriso dell'uomo/ che ha compreso. Anche il sole trascorre remoto/arrossando le spiagge. Verrà il giorno che il dio/ non saprà più dov'erano le spiagge d'un tempo». Anche se, nei Dialoghi, il poeta rilutterà a quest'altra specie di solitudine, tenterà di riallacciarsi, in stupori e affanni, al miracolo delle origini, al fanciullo «divino».

Quali che siano i risultati raggiunti nella disposizione dei materiali, nell'ambizione di fare racconto, non più all'interno di ogni singola poesia, ma attraverso le loro più o meno esplicite connessioni, Lavorare stanca ha tutta l'aria di essere per Pavese un libro definitivo. Se si tiene presente che, dopo il 1940, si dedicherà con trasporto soltanto alla narrativa (oltrechè alle note del diario). Ma tornerà a fare versi sotto l'empito, che si direbbe trascinante, di una nuova passione d'amore. Nel 1945 detta in pochi mesi le poesie di La terra e la morte il cui nerbo è ispirato dal suo legame con Bianca Garufi (mentre le poesie d'amore di Lavorare stanca avevano come referenti Tina Pizzardo e Fernanda Pivano). Escludiamo le due poesie che risentono, tra commozione e rimorso, della guerra civile ( $« \mathrm{Tu}$ non sai le colline/ dove si è sparso il sangue», «E allora noi vili/ che amavamo la sera») e trovano un ideale riscontro nelle pagine della Casa in collina. Le altre sette compongono incontestabilmente un acceso canzoniere amoroso in cui Pavese ricorre alle cadenze mosse e celeri del settenario. Esse si rivolgono in tono estatico alla sua donna, 
assunta come termine di paragone e di scambio con il mare, la collina, la vigna: «Terra rossa terra nera/ tu vieni dal mare,/ dal verde riarso,/ dove sono parole/ antiche e fatica sanguigna/ e gerani tra i sassi...».

A lei viene consegnato anche il paesaggio umano e morale delle Langhe: «il gesto del contadino», «la grande fatica», «le parole rassegnate sulle soglie,/ il grido del bimbo-le cose che non passano mai». È in questa scarna pronuncia, in questo catalogo di momenti mitici che si avverte l'eco superstite e terragna di Lavorare stanca. E l'invocazione a un «tu» che non ha una personale connotazione, l'incalzare del verbo al presente (sei...sei...; hai, hai...) la monotona litania suggeriscono l'intatta adesione del credente. Pavese, accennando a queste poesie, le definisce «quasi dannunziane». E davvero vien da pensare, per l'effuso colore d'estate, ai versi dell'Alcyone, in particolare a Novilunio. Dove troviamo analoghe iterazioni in lode della «creatura/ celeste che ha nome/ Luna», ma anche della «creatura/ terrestre che ha nome/ Ermione»: il Novilunio di settembre è «tiepido come/ le sue chiome,/ umido come il sorriso/ della sua bocca/ umida ancòra/ della prima uva matura,/ breve come la sua cintura/ nel cielo verde/ come la sua veste». E via seguitando. D'altronde è lo stesso poeta che, pronunciando un «quasi» limitativo, ci suggerisce di indagare sulle sue frequentazioni dannunziane. Ne troviamo qualche indizio anche in talune tessere lessicali presenti in La terra e la morte: la preziosa «conca del braciere», l'inusuale «vento che ti giunge».

Ho esordito - in questa rilettura abbandonata, aperta a emozioni e suggestioni di varia natura - dicendo che Pavese nasce poeta. Ma va ricordato anche che egli muore poeta. Alludo ovviamente alle 11 poesie di Verrà la morte e avrà i tuoi occhi, scritte nel marzo del 1950 e dedicate a Constance Dowling (due in inglese, che è la sua lingua). Ripetono in parte le invocazioni di La terra e la morte, ma si respira in esse un'aria diversa: là era il mondo mediterraneo, il Meridione a cui apparteneva Bianca; qui si parla di nevi gelate e vento di marzo, o di strade vuote nel primo mattino. I giorni di Cervinia e Torino, vissuti in compagnia di Connie, lasciano in queste poesie un'eco più raggelata e nordica. Fa eccezione Roma, dove Pavese corre all'ultimo appuntamento, dandone testimonianza in una fascinosa poesia ancora pervasa da un sentimento di giovanile fervore e fiducia Passerò per Piazza di Spagna: «S'aprirà quella strada,/ le pietre canteranno,/ il cuore batterà sussultando/ come l'acqua nelle fontane -/ sarà questa la voce/ che salirà le tue scale». Queste poesie si muovono dunque in direzioni diverse, facendo spazio alla città, e con diversi risultati espressivi, ubbidendo all'urgenza della passione. A questo punto non è più questione, per Pavese, di struttura o di canzoniere. Adesso ogni poesia vale inesorabilmente per sè: «Verrà la morte e avrà i tuoi occhi - / questa morte che ci accompagna/ dal mattino alla sera, insonne,/ sorda, come un vecchio rimorso/ o un vizio assurdo». Ogni mattina, fissandosi allo specchio, la donna amata potrà scoprire di essere portatrice e vittima insieme di un dono funesto. Nel disincanto estremo, dismesso ogni rancore e autocommiserazione, Pavese si apre, attraverso la figura di Connie, a una indistinta, universale condivisione: $« \mathrm{O}$ cara speranza,/ quel giorno sapremo anche noi/ che sei la vita e sei il nulla». Si mostra fedele, in modi più radicali e ultimativi, al testamento vergato nel Mestiere di vivere: «Ho lavorato, ho dato poesia agli uomini, ho condiviso le pene di molti». 


\section{Bibliografia}

BÀRBERI-SQUAROTTI, Giorgio (2001): «Da Verlaine a Pavese, carcere e simbolo», in Studi sulla letteratura in Val Bormida (e dintorni). Millesimo. Comunità Montana «Alta Val Bormida». Millesimo.

Bogliolo, Giovanni (1991): Cinquantadue trame di capolavori della letteratura francese dell'Ottocento. Milano, Rizzoli.

GINZBURG, Leone (2004): Lettere dal confino, a cura di Luisa Mangoni. Torino, Einaudi.

Mondo, Lorenzo (1964): «Fra Gozzano e Whitman le origini di Pavese», in Sigma, 3-4, pp. 3-21.

Mutterle, Anco M. (1987): «Da Gozzano a Pavese», in Cesare Pavese oggi. Atti del Convegno di San Salvatore Monferrato, 25-26-27, a cura di Giovanna Ioli. San Salvatore Monferrato, pp. 158-170.

PAVESE, Cesare (1943): «A proposito di alcune poesie non ancora scritte», in Appendice a Lavorare stanca. Torino. Einaudi.

PAVESE, Cesare (1961a): Poesie, prefazione di M. Mila. Torino, Einaudi.

PAVESE, Cesare (1961b): Scritti giovanili, Collocazione APX52, c.155, «Centro Studi Guido Gozzano-Cesare Pavese» dell'Università di Torino.

PAVESE, Cesare (1962): Poesie edite e inedite, a cura di Italo Calvino. Torino, Einaudi.

PAVESE, Cesare (1998): Tutte le poesie, a cura di Mariarosa Masoero. Torino, Einaudi.

PAVESE, Cesare (2008): Officina Einaudi, Lettere editoriali 1940-1950, a cura di Silvia Savioli e introduzione di Franco Contorbia. Torino, Einaudi.

RIPOSIO, Dino (1987): «Ipotesi sulla metrica di Lavorare stanca», in Cesare Pavese oggi, edit. pp. 41-46. 